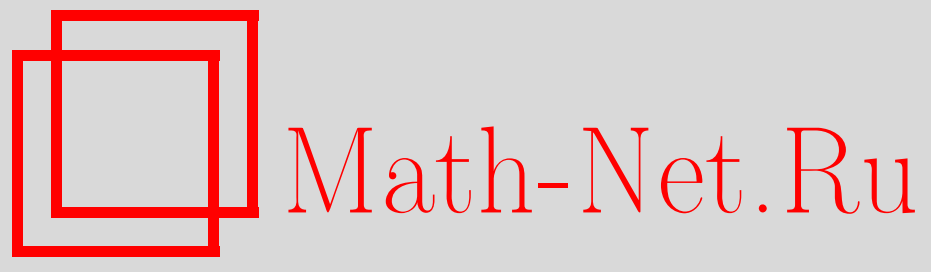

Г. М. Жислин, О стабильности $n$-частичных псевдорелятивистских систем, ТМФ, 2007, том 152, номер 3, 528537

DOI: https://doi.org/10.4213/tmf6108

Использование Общероссийского математического портала Math-Net.Ru подразумевает, что вы прочитали и согласны с пользовательским соглашением http://www . mathnet.ru/rus/agreement

Параметры загрузки:

IP : 54.164 .48 .24

26 апреля 2023 г., 17:53:41

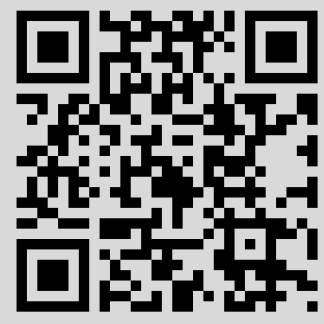




\title{
О СТАБИЛЬНОСТИ $n$-ЧАСТИЧНЫХ ПСЕВДОРЕЛЯТИВИСТСКИХ СИСТЕМ
}

\begin{abstract}
Для системы $Z_{n}$, состоящей из $n$ тождественных псевдорелятивистских частиц, показано, что при некоторых ограничениях на парные потенциалы взаимодействия существует бесконечная последовательность таких чисел $n_{s}, s=$ $1,2, \ldots$, что система $Z_{n}$ стабильна при $n=n_{s}$, причем $\sup _{s} n_{s+1} n_{s}^{-1}<+\infty$. Кроме того, показано, что если система $Z_{n}$ стабильна, то при некоторых значениях полного момента частиц системы дискретный спектр оператора энергии относительного движения $Z_{n}$ не пуст. Ранее устойчивость $n$-частичных систем изучалась только для нерелятивистских частиц.
\end{abstract}

Ключевые слова: псевдорелятивистские операторы, многочастичные системы, устойчивость, дискретный спектр.

\section{ВВЕДЕНИЕ}

В настоящей работе исследуется устойчивость системы $Z_{n}$, состоящей из $n$ тождественных псевдорелятивистских ${ }^{1)}$ (ПР) частиц, при $n \rightarrow \infty$ и выясняется связь между устойчивостью системы и существованием непустого дискретного спектра гамильтониана относительного движения. При некоторых условиях на потенциалы парного взаимодействия частиц мы доказываем существование такой последовательности "магических" чисел $n_{s}$, что система $Z_{n}$ устойчива при $n=n_{s}, s=1,2, \ldots$, и устанавливаем равномерную (по $s$ ) ограниченность отношения $n_{s+1} / n_{s}$ (теорема 1). Ранее аналогичный результат об устойчивости $n$-частичных систем был получен только для нерелятивистских (НР) частиц [1], а оценка величин $n_{s+1} / n_{s}$ отсутствовала даже в НР-случае. Что касается связи между устойчивостью системы и существованием непустого дискретного спектра оператора энергии относительного движения, то для НР-систем согласно теореме Хунцикера-Ван ВинтераЖислина (ХВЖ) [2], [3] из устойчивости системы следует существование хотя бы одного дискретного собственного значения соответствующего оператора при любых

\footnotetext{
1) Мы называем квантовую систему, составляющие ее частицы и соответствующий гамильтониан псевдорелятивистскими, если расматриваются релятивистские операторы кинетической энергии частиц и нерелятивистские операторы потенциальной энергии.
}

*Научно-исследовательский радиофизический институт, Нижний Новгород, Россия. E-mail: greg@nirfi.sci-nnov.ru 
значениях полного момента $P$ системы $Z_{n}$, в то время как для ПР-систем подобный результат установить не удается, скорее всего, он вообще не имеет места. Такая ситуация обусловлена тем, что ПР-оператор энергии $H_{n}(P)$ относительного движения (см. п. 1.4) зависит от полного момента $P$, а в НР-случае зависимость от $P$ отсутствует. Поэтому даже для устойчивых ПР-систем нам удается доказать, что дискретный спектр оператора $H_{n}(P)$ не пуст только для некоторых, но отнюдь не для всех значений $P$ (теорема 2).

\section{1. ОПРЕДЕЛЕНИЯ И ОСНОВНЫЕ РЕЗУЛЬТАТЫ}

1.1. Рассмотрим квантовую систему $Z_{n}=\{1,2, \ldots, n\}$, состоящую из $n$ тождественных частиц. Пусть $r_{i}=\left(x_{i}, y_{i}, z_{i}\right)$ и $m$ - радиус-вектор и масса $i$-й частицы, $r=\left(r_{1}, \ldots, r_{n}\right)$. ПР-гамильтониан системы $Z_{n}$ имеет вид

$$
H_{n}=K_{n}(r)+V_{n}(r),
$$

где $\mathrm{e}^{2)}$

$$
\begin{gathered}
K_{n}(r)=\sum_{i=1}^{n} K\left(r_{i}\right), \quad V_{n}(r)=\frac{1}{2} \sum_{\substack{i, j=1 \\
i \neq j}}^{n} V\left(\left|r_{i j}\right|\right), \\
K\left(r_{i}\right)=\sqrt{-\Delta_{i}+m^{2}}, \quad \Delta_{i}=\frac{\partial^{2}}{\partial x_{i}^{2}}+\frac{\partial^{2}}{\partial y_{i}^{2}}+\frac{\partial^{2}}{\partial z_{i}^{2}}, \quad r_{i j}=r_{i}-r_{j} .
\end{gathered}
$$

Мы предполагаем, что

$$
\begin{gathered}
V\left(\left|r_{1}\right|\right) \in \mathcal{L}_{2, \text { lос }}\left(\mathbb{R}^{3}\right), \\
V\left(\left|r_{1}\right|\right) \rightarrow 0 \quad \text { при } \quad\left|r_{1}\right| \rightarrow \infty^{3)}
\end{gathered}
$$

и потенциал $V\left(\left|r_{1}\right|\right)$ таков, что для некоторого $\varepsilon_{0}>0$

$$
\inf \left\{H_{n}+\varepsilon V_{n}\right\}>-\infty \quad \text { при } \quad 0 \leqslant \varepsilon \leqslant \varepsilon_{0} .
$$

Операторы $K\left(r_{i}\right)$ в координатном представлении являются интегральными операторами, но в импульсном представлении они представляют собой операторы умножения. Действительно, пусть $\mathcal{F}$ есть преобразование Фурье в $\mathbb{R}^{3}$ и $\bar{f}\left(p_{i}\right)=\mathcal{F}\left[f\left(r_{i}\right)\right]$ для любой функции $f\left(r_{i}\right) \in \mathcal{L}_{2}\left(\mathbb{R}^{3}\right)$, где $p_{i}$ - фурье-сопряженные координаты к $r_{i}$. Тогда

$$
\mathcal{F}\left[K\left(r_{i}\right) f\left(r_{i}\right)\right]=\mathcal{F}\left[\sqrt{-\Delta_{i}+m^{2}} f\left(r_{i}\right)\right]=\sqrt{p_{i}^{2}+m^{2}} \bar{f}\left(p_{i}\right) .
$$

Положим $p=\left(p_{1}, \ldots, p_{n}\right)$,

$$
\bar{K}\left(p_{i}\right)=\sqrt{p_{i}^{2}+m^{2}}, \quad \bar{K}_{n}(p)=\sum_{i=1}^{n} \bar{K}\left(p_{i}\right)
$$

и используем далее смешанную форму записи для оператора $H_{n}$ :

$$
H_{n}=\bar{K}_{n}(p)+V_{n}(r) .
$$

\footnotetext{
2) Система единиц выбрана так, что константа Планка и скорость света равны единице.

3) Требование (1.2б) может быть несколько ослаблено, но мы не останавливаемся на этом, так как (1.2б) выполняется для большинства интересных приложений.
}

5 Теоретическая и математическая физика, т. 152, № 3, 2007 г. 
1.2. Пусть $D_{k}=\left\{C_{1}, \ldots, C_{k}\right\}$ - произвольное разбиение системы $Z_{n}$ на $k$ подсистем $C_{j}$, не имеющих общих элементов и не взаимодействующих друг с другом, $H_{n}\left(D_{k}\right)$ - оператор энергии составной системы $D_{k}$. Очевидно,

$$
H_{n}\left(D_{k}\right)=\sum_{j=1}^{k} H\left[C_{j}\right]
$$

где

$$
H\left[C_{j}\right]=\sum_{i \in C_{j}} \bar{K}\left(p_{i}\right)+\frac{1}{2} \sum_{\substack{s, t \in C_{j} \\ s \neq t}} V\left(\left|r_{s t}\right|\right) .
$$

В силу (1.3) операторы $H_{n}$ и $H_{n}\left(D_{k}\right)$ ограничены снизу на $C_{0}^{2}\left(\mathbb{R}^{3 n}\right), \mathbb{R}^{3 n}=\{r=$ $\left.\left(r_{1}, \ldots, r_{n}\right)\right\}$. Мы расширим их (по Фридрихсу) до самосопряженных операторов, сохраняя прежние обозначения. Нетрудно показать, что для любых разбиений $D_{k}$

$$
\inf H_{n} \leqslant \inf H_{n}\left(D_{k}\right)=\sum_{i=1}^{k} \inf H\left[C_{i}\right] .
$$

1.3. В настоящей работе исследуется вопрос о стабильности ПР-системы $Z_{n}$ при $n \rightarrow \infty$. Учитывая физический смысл операторов $H_{n}$ и $H_{n}\left(D_{k}\right)$ (это операторы энергии), мы назовем систему $Z_{n}$ устойчивой, если

$$
\inf H_{n}<\mu_{n}:=\min _{D_{k}} \inf H_{n}\left(D_{k}\right), \quad 2 \leqslant k \leqslant n
$$

Возможны два варианта поведения системы $Z_{n}$ :

1) существует такое $N>0$, что система $Z_{n}$ неустойчива при всех $n \geqslant N$;

2) для любого $N>0$ мы можем указать такое $n>N$, что система $Z_{n}$ устойчива.

Естественно, поведение системы $Z_{n}$ определяется свойствами потенциалов $V\left(\left|r_{1}\right|\right)$. Наше рассмотрение включает такие взаимодействия $V\left(\left|r_{1}\right|\right)$, для которых априори не ясно, какой из двух названных вариантов реализуется. Например, наши результаты применимы к знакопеременным потенциалам, которые представляют собой разность двух потенциалов Юкавы:

$$
V\left(\left|r_{1}\right|\right)=W\left(\left|r_{1}\right|\right)=\left|r_{1}\right|^{-1}\left(a_{1} e^{-b_{1}\left|r_{1}\right|}-a_{2} e^{-b_{2}\left|r_{1}\right|}\right),
$$

где $a_{1}>a_{2}>0, b_{1}>b_{2}>0$.

Обозначим через $K(A)$ куб в $\mathbb{R}^{3}$ с ребром длины $2 A$, центром в начале координат и гранями, параллельными координатным плоскостям. Нашим главным результатом является следующая

Tеорема 1. Пусть для некоторого $A>0$

$$
\delta=\int_{r_{1}, r_{2} \in K(A)} V\left(\left|r_{1}-r_{2}\right|\right) d r_{1} d r_{2}<0 .
$$

Тогда 
а) существует последовательность таких чисел $n_{1}<n_{2}<\cdots<n_{s}<\cdots$, где $n_{s} \rightarrow \infty$ при $s \rightarrow \infty$, что системы $Z_{n_{s}}, s=1,2, \ldots$, устойчивы;

б) если потенциаль $V(|t|)$ ограничены снизу, то для чисел $n_{s}$ выполняется оценкa

$$
\sup _{s} \frac{n_{s+1}}{n_{s}}<+\infty
$$

ЗАмечАниЕ 1. Для выполнения неравенства (1.8) достаточно, чтобы были выполнены условие $V\left(\left|r_{1}\right|\right) \in \mathcal{L}_{1}\left(\mathbb{R}^{3}\right)$ и неравенство

$$
\int_{\mathbb{R}^{3}} V\left(\left|r_{1}\right|\right) d r_{1}<0
$$

(см. [1]). Для оператора $W$ из (1.7) неравенство (1.10) выполняется, если

$$
a_{1} a_{2}^{-1}<b_{1}^{2} b_{2}^{-2}
$$

1.4. Обсудим теперь связь между устойчивостью системы $Z_{n}$ и существованием непустого дискретного спектра ПР-оператора энергии относительного движения $Z_{n}$. Для НР-систем согласно теореме ХВЖ [2], [3] из устойчивости системы следует существование непустого дискретного спектра оператора энергии относительного движения независимо от значения $P$ полного момента частиц системы. Независимость от $P$ возникает после отделения движения центра масс, которое проводится в НР-системах для получения оператора энергии относительного движения. Для ПР-операторов такое отделение невозможно, в результате чего ПР-оператор энергии относительного движения зависит от значения $P$ полного момента частиц системы $Z_{n}$ [4]. Именно поэтому связь устойчивости системы $Z_{n}$ с существованием непустого дискретного спектра соответствующего оператора в ПР-случае сложнее, чем для НР-систем. Чтобы сформулировать полученные результаты, построим (следуя [4]) ПР-оператор энергии относительного движения системы $Z_{n}$. Введем радиус-вектор $r_{0}=\sum_{j=1}^{n} r_{j} / n$ центра масс системы $Z_{n}$ и относительные координаты $q_{j}=r_{j}-r_{0}, j=1,2, \ldots, n$, частиц системы $Z_{n}$. Фурье-сопряженные координаты для $q_{j}$ суть те же $p_{j}$, что для $r_{j}$, а для координаты $r_{0}$ сопряженная координата есть $P=\sum_{j=1}^{n} p_{j} \in \mathbb{R}^{3}$, что дает значение полного импульса частиц системы $Z_{n}$. Легко видеть, что после введения в $H_{n}$ новых координат $r_{0}, q_{j}, j=1, \ldots, n\left(P\right.$ и $p_{j}$, $j=1,2, \ldots, n$, для оператора $\bar{K}_{n}(p)$ кинетической энергии), мы получим оператор $H_{n}(P)$, который будет зависеть от относительных координат $q_{j}$, соответствующих моментов $p_{j}$ и полного момента $P$, но не будет зависеть от положения $r_{0}$ центра масс. Поэтому, если фиксировать произвольное значение $P \in \mathbb{R}^{3}$ и рассматривать полученный оператор на функциях $\varphi\left(q_{1}, \ldots, q_{n}\right)$ с условием $\sum_{j=1}^{n} q_{j}=0$ (на функциях $\bar{\varphi}\left(p_{1}, \ldots, p_{n}\right)$ с условием $\sum_{j=1}^{n} p_{j}=P$ для оператора кинетической энергии), то такой оператор можно интерпретировать как оператор энергии относительного движения системы с фиксированным значением $P$ полного момента. Для оператора $H_{n}(P)$ имеет место

ТеОРема 2. Если система $Z_{n}$ устойчива, то существует конечное или счетное число таких открытых областей $\gamma_{\alpha} \subset \mathbb{R}^{3}$, что дискретный спектр оператора $H_{n}(P)$ не пуст при $P \in \gamma_{\alpha}$. 
ЗАмЕчАНИЕ 2. К сожалению, мы не можем указать явный вид областей $\gamma_{\alpha}$ (хотя и описываем, как они получаются) и не знаем, например, выполняется ли равенство $\bigcup_{\alpha} \gamma_{\alpha}=\mathbb{R}^{3}$. Это связано с тем, что зависимость спектра операторов $H_{n}(P)$ и $H_{n}\left(P ; D_{k}\right)$ от $P$ при $n>2$ до сих пор не изучена (случай $n=2$ исследован в [5], [6]).

\section{2. ДОКАЗАТЕЛЬСТВА ТЕОРЕМ}

2.1. Доказательство теоремы 1 проведем от противного. Предположим, что система $Z_{n}$ неустойчива при всех $n \geqslant N$ для какого-либо $N>0$,

и докажем, что это предположение приводит к неравенству

$$
\inf H_{n} \geqslant-d_{1} n
$$

для всех $n \geqslant N$, и некоторой константы $d_{1}>0$, не зависящей от $n$. После этого мы построим такие пробные функции $\psi_{n},\left\|\psi_{n}\right\|=1$, что для больших $n$

$$
\left(H_{n} \psi_{n}, \psi_{n}\right) \leqslant-d_{2} n^{2}
$$

где $d_{2}>0$ - константа, не зависящая от $n$. Тогда из $(2.1),(2.2)$ будет следовать, что

$$
d_{1} n \leqslant-d_{2} n^{2}
$$

откуда в пределе при $n \rightarrow \infty$ получаем неравенство $-d_{2} \geqslant 0$, которое неверно, поскольку $d_{2}>0$. Поэтому предположение $(*)$ ошибочно, откуда и будет следовать утверждение теоремы 1.

2.2. Пусть предположение $(*)$ выполняется и $n \geqslant N$. Тогда система $Z_{n}$ неустойчива, и, следовательно, существует такое разбиение $D_{k}=\left\{C_{1}, \ldots, C_{k}\right\}$ системы $Z_{n}$, что

$$
\inf H_{n}=\inf H_{n}\left(D_{k}\right)=\sum_{j=1}^{k} \inf H\left[C_{j}\right] .
$$

Выберем разбиение $D_{k}$ так, что каждый кластер $C_{j}$ из $D_{k}$ устойчив. Это можно сделать, ибо если какой-то кластер $C_{i}$ неустойчив, тогда (согласно определению п. 1.3 , см. условие (1.6), примененному не ко всей системе $Z_{n}$, а к кластеру $C_{i}$ ) мы можем разбить множество $C_{i}$ на непересекающиеся подмножества $C_{i t}, t=1,2, \ldots, t_{i}$, $t_{i} \geqslant 2($ !) так, что

$$
\inf H\left[C_{i}\right]=\sum_{t=1}^{t_{i}} \inf H\left[C_{i t}\right]
$$

и заменить разбиение $D_{k}$ на разбиение

$$
\widetilde{D}_{k-1+t_{i}}=\left\{C_{1}, \ldots, C_{i-1}, C_{i 1}, \ldots, C_{i t_{i}}, C_{i+1}, \ldots, C_{k}\right\},
$$

для которого

$$
\inf H_{n}=\inf H_{n}\left(\widetilde{D}_{k-1+t_{i}}\right)=\sum_{j, j \neq i}^{1, k} \inf H\left[C_{j}\right]+\sum_{t=1}^{t_{i}} \inf H\left[C_{i t}\right] .
$$


Если разбиение $\widetilde{D}_{k-1+t_{i}}$ содержит неустойчивые кластеры $\left(C_{j}\right.$ или $\left.C_{i t}\right)$, то каждый из них можно заменить некоторым набором составляющих его кластеров подобно тому, как мы поступили с кластером $C_{i}$. Продолжая этот процесс, мы придем в итоге к такому разбиению, в котором нет неустойчивых кластеров. Поэтому можно считать, что с самого начала взято именно такое разбиение $D_{k}$, в котором все кластеры устойчивы.

Согласно предположению $(*)$ число $|C|$ элементов устойчивого кластера $C$ не превышает $N$. Поэтому, если положить

$$
d=\min _{C,|C| \leqslant N} \inf H[C], \quad C \subset Z_{n},
$$

и $d_{1}=|d|$, то в силу $(2.3)$

$$
\inf H_{n} \geqslant-d_{1} n \text {. }
$$

Неравенство (2.1) доказано.

2.3. Переходим к доказательству неравенства (2.2). Пусть число $A>0$ выбрано так, что выполняется неравенство (1.8). Для любого целого $\omega>0$ определим нормированные функции

$$
f_{p}\left(x_{1}\right)=\left\{\begin{array}{lll}
A^{-1 / 2} \sin \left(\frac{2 \pi p \omega}{A} x_{1}\right) & \text { при } & \left|x_{1}\right| \leqslant A, \\
0 & \text { при } & \left|x_{1}\right|>A
\end{array}\right.
$$

И

$$
f_{i j k}\left(r_{s}\right)=f_{i}\left(x_{s}\right) f_{j}\left(y_{s}\right) f_{k}\left(z_{s}\right), \quad s=1,2, \ldots, n .
$$

Для фиксированного $n$ укажем целое число $n_{0}$ так, что $n_{0}^{3} \leqslant n<\left(n_{0}+1\right)^{3}$ и положим

$$
\varphi_{t}\left(r_{t}\right)=f_{i_{t} j_{t} k_{t}}\left(r_{t}\right), \quad \psi_{n}(r)=\prod_{t=1}^{n} \varphi_{t}\left(r_{t}\right),
$$

где $i_{t}, j_{t}, k_{t}$ - натуральные числа, $1 \leqslant i_{t}, j_{t}, k_{t} \leqslant n_{0}+1,\left(i_{t}, j_{t}, k_{t}\right) \neq\left(i_{s}, j_{s}, k_{s}\right)$ при $t \neq s$.

Покажем, что для функций $\psi(r)$ выполняется неравенство (2.2). Сначала оценим формы $\left(K_{n}(r) \psi_{n}, \psi_{n}\right)=\left(\bar{K}_{n}(p) \bar{\psi}_{n}, \bar{\psi}_{n}\right)$,

$$
\left(K_{n}(r) \psi_{n}, \psi_{n}\right)=\sum_{t=1}^{n}\left(K\left(r_{t}\right) \psi_{n}, \psi_{n}\right),
$$

где в силу ортонормированности функций $\varphi_{s}\left(r_{s}\right)$

$$
\begin{aligned}
\left(K\left(r_{t}\right) \psi_{n}, \psi_{n}\right) & \equiv\left(\sqrt{-\Delta_{t}+m^{2}} \psi_{n}, \psi_{n}\right)=\left(\sqrt{-\Delta_{t}+m^{2}} \varphi_{t}\left(r_{t}\right), \varphi_{t}\left(r_{t}\right)\right)_{\mathbb{R}^{3}\left(r_{t}\right)}= \\
& =\left(\sqrt{p_{t}^{2}+m^{2}} \bar{\varphi}_{t}\left(p_{t}\right), \bar{\varphi}_{t}\left(p_{t}\right)\right)_{\mathbb{R}^{3}\left(p_{t}\right)},
\end{aligned}
$$

$\bar{\varphi}_{t}\left(p_{t}\right)$ - фурье-образ функции $\varphi_{t}\left(r_{t}\right)$. 
Очевидно, $\sqrt{p_{t}^{2}+m^{2}} \leqslant m+p_{t}^{2}+1$, следовательно,

$$
\left(K\left(r_{t}\right) \psi_{n}, \psi_{n}\right) \leqslant m+1+\left\|\left|p_{t}\right| \bar{\varphi}_{t}\left(p_{t}\right)\right\|^{2}=m+1+\left\|\left|\nabla_{t} \varphi_{t}\left(r_{t}\right)\right|\right\|_{\mathbb{R}^{3}\left(r_{t}\right)}^{2} .
$$

Легко видеть, что

$$
m+1+\left\|\left|\nabla_{t} \varphi_{t}\left(r_{t}\right)\right|\right\|^{2} \leqslant c_{1}\left(n_{0}+1\right)^{2} \leqslant c_{2} n^{2 / 3},
$$

где $c_{1}, c_{2}$ - некоторые константы, зависящие от $A, \omega$, но не от $n$. В силу $(2.5)-(2.8)$

$$
\left(K_{n}(r) \psi_{n}, \psi_{n}\right) \leqslant c_{2} n^{5 / 3}
$$

2.4. Оценим теперь форму

$$
J(\omega)=\left(V_{n} \psi_{n}, \psi_{n}\right)=\sum_{\substack{s, t=1 \\ s<t}}^{n}\left(V\left(\left|r_{s t}\right|\right) \psi_{n}, \psi_{n}\right) .
$$

Докажем, что для некоторой константы $b>0$ и больших $n$

$$
J_{s t}(\omega)=\left(V\left(\left|r_{s t}\right|\right) \psi_{n}, \psi_{n}\right)<-b, \quad s, t=1,2, \ldots, n,
$$

если число $\omega$ взять достаточно большим.

Из (2.10), (2.11) будет следовать, что

$$
\left(V_{n} \psi_{n}, \psi_{n}\right) \leqslant-\frac{(n-1) n}{2} b \leqslant-\frac{n^{2}}{4} b
$$

Тогда соотношения (2.9), (2.12) приводят к требуемой оценке (2.2), и, тем самым, первое утверждение теоремы 1 доказано.

Очевидно, что

$$
J_{s t}(\omega)=\int_{r_{s}, r_{t} \in K(A)} V\left(\left|r_{s t}\right|\right) f_{i_{s} j_{s} k_{s}}^{2}\left(r_{s}\right) f_{i_{t} j_{t} k_{t}}^{2}\left(r_{t}\right) d r_{t} d r_{s}
$$

Так как $f_{p}^{2}(x)=(2 A)^{-1}\left(1-\cos \left(\frac{4 \pi \omega p}{A} x\right)\right)$, то мы можем записать $J_{s t}(\omega)$ в виде

$$
J_{s t}(\omega)=\sum_{\sigma_{1}, \ldots, \sigma_{6}}^{0,1} B_{\sigma_{1}, \ldots, \sigma_{6}}(\omega)
$$

где

$B_{\sigma_{1}, \ldots, \sigma_{6}}(\omega)=(2 A)^{-6} \int_{\xi, \xi^{\prime} \in K(A)} V\left(\left|\xi-\xi^{\prime}\right|\right) \prod_{p=1}^{6}(-1)^{\sigma_{p}} \cos ^{\sigma_{p}}\left(\frac{4 \pi \omega}{A} m_{p} \xi_{p}\right) d \xi_{1} d \xi_{2} \ldots d \xi_{6}$,

$\xi=\left(\xi_{1}, \xi_{2}, \xi_{3}\right), \xi^{\prime}=\left(\xi_{4}, \xi_{5}, \xi_{6}\right) \in \mathbb{R}^{3}, m_{p}$ - целое число, зависящее от $s, t$ и $n$, но не от $\omega$. 
2.5. Оценим величины $B_{\sigma_{1}, \ldots, \sigma_{6}}(\omega)$. Фиксируем произвольный набор $\sigma_{1}, \ldots, \sigma_{6}$ и положим для него

$$
l=\#\left\{\sigma_{j}, 1 \leqslant j \leqslant 6 \mid \sigma_{j}=1\right\} .
$$

При $l=0$ выполняется $\sigma_{1}=\sigma_{2}=\cdots=\sigma_{6}=0$ и по условию теоремы 1

$$
B_{0,0, \ldots, 0}(\omega)=(2 A)^{-6} \delta<0 .
$$

Рассмотрим случай $l \geqslant 1$ и покажем, что в этом случае

$$
B_{\sigma_{1}, \ldots, \sigma_{6}}(\omega) \rightarrow 0 \quad \text { при } \quad \omega \rightarrow \infty .
$$

Только чтобы упростить обозначения считаем, что в наборе $\sigma_{1}, \ldots, \sigma_{6}$ индексы $j$, для которых $\sigma_{j}=1$, суть $j=1,2, \ldots, l$.

Положим

$$
\Phi\left(\xi_{1}, \ldots, \xi_{l}\right)=(2 A)^{-6}\left\{\begin{array}{lll}
\int_{\substack{\left|\xi_{j}\right| \leqslant A \\
j=l+1, \ldots, 6}} V\left(\left|\xi-\xi^{\prime}\right|\right) d \xi_{l+1} \ldots d \xi_{6}, & \text { если } & 1 \leqslant l \leqslant 5 \\
V\left(\left|\xi-\xi^{\prime}\right|\right), & \text { если } & l=6 .
\end{array}\right.
$$

Тогда числа $B_{\sigma_{1}, \ldots, \sigma_{6}}(\omega)$ (см. (2.15)) можно рассматривать как фурье-коэффициенты функции $\Phi\left(\xi_{1}, \ldots, \xi_{l}\right)$. Функция $\Phi\left(\xi_{1}, \ldots, \xi_{l}\right) \in \mathcal{L}_{2, \text { loc }}\left(\mathbb{R}^{l}\right)$ (ибо $V(|\xi|) \in \mathcal{L}_{2, \text { loc }}\left(\mathbb{R}^{3}\right)$ ), поэтому (2.17) верно.

Из (2.16) и (2.17) следует, что при больших $\omega$ выполняется неравенство (2.11). Таким образом, второе утверждение теоремы 1 доказано.

2.6. Из доказанного утверждения следует существование бесконечной серии таких значений $n_{s}, s=1,2, \ldots, n_{s} \rightarrow+\infty$ при $s \rightarrow \infty$, что система $Z_{n}$ устойчива при $n=n_{s}, s=1,2, \ldots$.

Оценим отношение $n_{s+1} / n_{s}$. Пусть $k=n_{s+1}-1$. Не ограничивая общности, считаем, что $n_{s}<n_{s+1}-1$, следовательно, система $Z_{k}$ неустойчива. Рассуждая так же, как в п. 2.2 , убеждаемся, что найдется такое разбиение $\widetilde{D}=\left\{\widetilde{C}_{1}, \ldots, \widetilde{C}_{p}\right\}$ на устойчивые кластеры $\widetilde{C}_{j},\left|\widetilde{C}_{j}\right| \leqslant n_{s}{ }^{4)}$, что

$$
\inf H_{k}=\inf H_{k}(\widetilde{D})=\sum_{j=1}^{p} \inf H\left[\widetilde{C}_{j}\right]
$$

Поскольку потенциалы $V\left(\left|r_{1}\right|\right)$ ограничены снизу, то, очевидно,

$$
\inf H\left[\widetilde{C}_{j}\right] \geqslant-d_{0}\left|\widetilde{C}_{j}\right|^{2},
$$

откуда

$$
\inf H_{k} \geqslant-d_{0} \sum_{j=1}^{p}\left|\widetilde{C}_{j}\right|^{2}
$$

\footnotetext{
4)Напоминаем, что $\left|\widetilde{C}_{j}\right|$ - число элементов кластера $\widetilde{C}_{j}$.
} 
где

$$
\sum_{j=1}^{p}\left|\widetilde{C}_{j}\right|=k, \quad 1 \leqslant\left|\widetilde{C}_{j}\right| \leqslant n_{s}, \quad j=1,2, \ldots, p,
$$

и константа $d_{0}>0$ не зависит от $k$ и $n_{s}$.

Минимизируя правую часть (2.18) с учетом условий (2.19) и неравенства $d_{0}>0$, убеждаемся, что inf $H_{k} \geqslant-k n_{s} d_{0}$. С другой стороны, в силу (2.2) inf $H_{k} \leqslant-d_{2} k^{2}$, следовательно, $-d_{2} n_{s+1} \geqslant-n_{s} d_{0}$, т.е. $n_{s+1} n_{s}^{-1}<d_{0} d_{2}^{-1}$ при любых $s$.

Теорема 1 доказана полностью.

2.7. Доказательство теоремы 2. Пусть $D_{s}=\left\{C_{1}, \ldots, C_{s}\right\}-$ произвольное разбиение исходной системы $Z_{n}$ на непересекающиеся непустые кластеры $C_{j}, j=$ $1,2, \ldots, s$, и $H_{n}\left(P ; D_{s}\right)$ есть оператор $H_{n}\left(D_{s}\right)$ (см. п. 1.2) после введения относительных координат (см. п. 1.4) и фиксации значения $P$ полного момента системы $Z_{n}$ $\left(H_{n}\left(P ; D_{s}\right)\right.$ получается из $H_{n}\left(D_{s}\right)$ так же, как $H_{n}(P)$ получался из $\left.H_{n}{ }^{5)}\right)$. Пусть

$$
\mu_{n}(P)=\min _{D_{s}} \inf H_{n}\left(P ; D_{s}\right), \quad s \geqslant 2 .
$$

Согласно релятивистской теореме ХВЖ [4] $\mu_{n}(P)$ есть нижняя грань существенного спектра оператора $H_{n}(P)$. Поэтому, чтобы доказать теорему 2 , достаточно установить существование такого $P_{0} \in \mathbb{R}^{3}$, для которого

$$
\inf H_{n}\left(P_{0}\right)<\mu_{n}\left(P_{0}\right) \text {. }
$$

Легко видеть, что

$$
\inf H_{n}=\inf _{P} H_{n}(P) \text {. }
$$

Так как $\inf H_{n}(P)$ есть непрерывная функция от $P$ и

$$
\inf H_{n}(P) \rightarrow+\infty \quad \text { при } \quad|P| \rightarrow \infty,
$$

то в силу (2.21) существует по крайней мере одна точка $P_{0} \in \mathbb{R}^{3}$, для которой

$$
\inf H_{n}=\inf H_{n}\left(P_{0}\right) \text {. }
$$

Так как система $Z_{n}$ устойчива, то

$$
\inf H_{n}=\inf H_{n}\left(P_{0}\right)<\min _{D_{s}} \inf H_{n}\left(D_{s}\right),
$$

следовательно, для любого разбиения $D_{s}$

$$
\inf H_{n}\left(P_{0}\right)<\inf H_{n}\left(D_{s}\right) .
$$

Так же, как в случае оператора $H_{n}$, имеем

$$
\inf H_{n}\left(D_{s}\right)=\min _{P} \inf H_{n}\left(P ; D_{s}\right)
$$

\footnotetext{
5) Фактически $H_{n}\left(P ; D_{s}\right)$ - это оператор $H_{n}(P)$ после удаления из него всех членов взаимодействия между собой частиц из разных кластеров системы $D_{s}$.
} 
и в силу $(2.23),(2.24)$

$$
\inf H_{n}\left(P_{0}\right)<\inf H_{n}\left(P ; D_{s}\right)
$$

для любых $P$ и $D_{s}$. Из неравенства (2.25) следует $(2.20)$, и значит, дискретный спектр $s_{\mathrm{d}}\left(H_{n}(P)\right)$ оператора $H_{n}(P)$ не пуст при $P=P_{0}$. Далее, так как оператор $H_{n}(P)$ зависит от $P$ непрерывно, то из (2.25) следует существование такой открытой области $\gamma_{0} \subset \mathbb{R}^{3}$, что $s_{\mathrm{d}}\left(H_{0}(P)\right) \neq \varnothing$ при $P \in \gamma_{0}$.

Кроме того, если наряду с точкой $P_{0}$ существуют какие-либо точки $P_{\alpha}$ с тем же свойством $(2.22)$, что и $P_{0}$, т.е.

$$
\inf H_{n}=\inf H_{n}\left(P_{\alpha}\right) \text {, }
$$

то для каждой из них, очевидно, найдется такое открытое множество $\gamma_{\alpha}$, что

$$
s_{\mathrm{d}}\left(H_{n}(P)\right) \neq \varnothing, \quad P \in \gamma_{\alpha} .
$$

Теорема 2 доказана.

Благодарности. Работа поддержана РФФИ (грант № 05-01-00299).

\section{Список литературы}

[1] С. А. Вугальтер, Г. М. Жислин, ТМФ, 76 (1988), 132.

[2] Г. М. Жислин, ДАН СССР, 128 (1959), 231.

[3] М. Рид, Б. Саймон, Методы современной математической физики. Т. 4. Анализ операторов, Мир, М., 1982.

[4] R. T. Lewis, H. Siedentop, S. A. Vugalter, Ann. Inst. H. Poincaré Phys. Théor., 67 (1997), 1.

[5] С. А. Вугальтер, Г. М. Жислин, ТМФ, 121 (1999), 297.

[6] S. A. Vugalter, T. Weidl, Ann. Henri Poincaré, 4 (2003), 301.

Поступила в редакцию 16.11.2006 